\title{
Nueva normativa, para edificios mejor protegidos contra el fuego
}

\section{New regulations, towards better fire-protected buildings}

Rubén Benedetti

Centro de Desarrollo Tecnológico en Salud y Seguridad Edilicia (CDT SaSEd), Facultad de Arquitectura, Planeamiento y Diseño (FAPyD), Universidad Nacional de Rosario. Rosario,

Argentina

ruben.benedetti@unr.edu.ar

\section{RESUMEN}

En la Argentina existe un notable vacío normativo en lo que hace a la protección contra incendios. A falta de legislación específica se aplican las consideraciones del Decreto 351/79 que fue pensado como norma aplicable a la seguridad laboral. Cuatro décadas después, sin haber sido actualizado, se mantienen fuera de las prescripciones legales elementos básicos como las montantes secas, o sistemas innovadores como las nieblas a alta presión. La falta de normas se hace extensiva a los servicios de bomberos y a la provisión de agua para extinción, dando lugar a condiciones muy poco favorables para las operaciones de lucha contra el fuego. El trabajo examina los conflictos debidos a esta situación y propone fuentes para la actualización de normas aplicables.

\section{ABSTRACT}

In Argentina there is a noticeable regulatory void in terms of Fire Protection. In the absence of specific legislation, the provitions of Decree $351 / 79$ are widely applied, despite this was originally intended as a standard applicable to occupational safety. Four decades later, without having been updated, basic elements such as dry standpipes, or innovative systems such as high pressure mists, remain outside the legal requirements. The lack of regulations extends to fire services and the provision of water for fire services, resulting in very poor conditions for fire fighting operations. This work considers the conflicts due to this situation and proposes sources for updating applicable standards.

PALABRAS CLAVE: seguridad, incendios, legislación.

KEY WORDS: security, fire, legislation.

FECHA DE RECEPCIÓN: 31/08/2020 | FECHA DE ACEPTACIÓN: 12/11/2020

DOI: http://dx.doi.org/10.30972/arq.0164556 
Es evidente que el incendio es uno de los principales riesgos que enfrentan los edificios. Junto con los riesgos de derrumbe ocasionados por sismos, constituyen la primera amenaza a la integridad de cualquier construcción. Pero no todos los edificios están en zona sísmica, e incluso los edificios sismorresistentes corren riesgo de sufrir daños irreparables en virtud de incendios que sigan a un terremoto.

A la ocurrencia de incendios accidentales se suma la posibilidad de fuegos intencionales ocasionado por acciones delictivas o actos terroristas, lo cual hace que la posibilidad de que un edificio resulte destruido o con daños que lo inhabilitan por largo tiempo es mucho mayor que la ocurrencia de una catástrofe natural.

En los últimos años, en nuestro medio se puede verificar un incremento en los incendios tanto en edificios de vivienda como en instalaciones comerciales e industriales, lo que encuentra como explicación a priori el aumento de las instalaciones energizadas, usos mixtos cada vez más complejos; la falta de actualización de tendidos eléctricos internos, y por supuesto la ausencia de instalaciones de detección y extinción adecuadas. Sin embargo, a pesar de este incremento en los siniestros con daños mayores y víctimas en la Argentina no se han modificado mayormente las exigencias en la prevención ni en edificios nuevos ni en edificios existentes; por otra parte, es generalizada- salvo escasas excepciones- la escasez en la provisión del insumo indispensable para atacar incendios: el agua.

Con la excepción de algunas pocas ciudades donde se han elaborado normas municipales más avanzadas, las disposiciones aplicables en materia de prevención de incendio corresponden a lo establecido por el decreto 351, reglamentario de la ley 19.587 de Higiene y Seguridad. (R.A., 1979). Es decir, que a pesar del notable salto tecnológico ocurrido en las últimas décadas, en las que se han incorporado al hábitat humano numerosos equipos de climatización, informáticos, de comunicaciones, y de confort, seguimos aplicando las prescripciones de una norma que ya superó los 40 años, y que en última instancia tuvo como objeto determinar las condiciones de seguridad edilicia exigibles en establecimientos laborales, una norma que ante la falta de disposiciones específicas al respecto se ha convertido de hecho en una suerte de norma patrón, para el proyecto de edificios de vivienda, centros comerciales, lugares de asamblea, templos, y todo el amplio abanico edilicio que compone el hábitat, una circunstancia que aunque no establecida en la letra de la norma parece haber sido considerada en la redacción al incluir en los cuadros la categoría "vivienda- residencia colectiva" o "actividades religiosas", que parecen alejadas de la definición de establecimiento laboral.

Como muestra del añejamiento normativo aludido, cabe detenerse en los usos definidos en el cuadro de factores de ocupación (apartado 3.1.2 del Anexo VII) donde se mencionan algunos tan arcaicos como "salas de baile"; "salones de billares", "canchas de bochas"; o en el cuadro de protección contra incendio (apartado 7.2 del Anexo VII) cuando se alude a garajes con expendio de combustible, una tipología edilicia que probablemente ya estaba extinta cuando se sancionó el decreto.

El caso es que, con todas sus falencias, el vetusto 351 continúa siendo la norma general de aplicación en el país, y en los casos en que reglamentaciones municipales han abordado el tema de la prevención de incendios y la evacuación, como regla general las normas locales han calcado las prescripciones, los valores, e incluso la tipificación de usos del Decreto.

Una evidencia absurda de este calco aparece en la sección en que el decreto en su artículo 160 define como autoridad técnica respecto a instalaciones contra incendio a la Superintendencia de Bomberos de la Policía Federal una disposición de por sí cuestionable, tratándose de una norma de alcance nacional, al designar a un organismo que fuera de la Capital Federal nunca tuvo jurisdicción en el interior, con excepción de algunos destacamentos aeroportuarios. El caso es que esta circunstancia, en numerosas normas municipales se tradujo en que se designara como autoridad en la materia a los cuerpos de bomberos locales, cuando en la mayor parte del país los bomberos son cuerpos voluntarios con escasa o ninguna formación específica en seguridad 
edilicia. Para el caso, puede citarse el ejemplo de la ciudad de Rosario, donde su Reglamento Municipal (Sección 3.10.) establece la autoridad de "la Dirección de bomberos de la Policía", siendo que el Cuerpo de Bomberos Zapadores, dependiente de la Policía de la Provincia de Santa Fe no tiene orgánicamente ninguna formación para entender en materia de riesgos edilicios, careciendo de la profunda formación técnica que caracterizó a la Superintendencia Federal de Bomberos.

Algunos intentos de actualización operados sobre las normas municipales han dado, incluso, resultados peores. En un intento de incorporar soluciones avanzadas, se ha hecho uso del copiar y pegar de fuentes normativas diversas, dando como resultado paradójico que la norma municipal no condice con la norma nacional implicando un verdadero intríngulis para el proyectista que debe definir si prioriza las exigencias para la habilitación local o los requerimientos que en caso de establecimientos laborales verificará la aseguradora de riesgos del trabajo e inspeccionará la Autoridad de Trabajo Local.

Volviendo al decreto 351 , en cuanto a las normas técnica aplicables para selección de materiales, características técnicas de las instalaciones, métodos de dimensionamiento e incluso procedimientos de ensayo, se refiere en el mismo a la "normas y reglamentaciones vigentes y las dictadas o a dictarse por la Superintendencia de Bomberos de la Policía Federal". De más está decir que ese cuerpo no emitió nunca normas de difusión general sobre estos temas, y si existió alguna norma interna, su falta de difusión equivale a una suerte de desuetudo automático. Resulta así que la norma nacional requería para las instalaciones contra incendio apegarse a unos requerimientos técnicos inexistentes, de manera que, en suma, todas sus especificaciones sobre instalaciones contra incendio son apenas un conjunto de lineamientos básicos sin especificaciones claras.

Merece una aclaración que la creación del Sistema de Riesgos del Trabajo, no aporto nada sobre la materia, lo cual en cierto modo tiene sentido por cuanto el problema excede largamente el mundo laboral. Sin embargo, fue el IRAM desde inicios de la década del 2000 , que reconociendo el vacío existente en la materia dictó una importante serie de normas, aplicables a las instalaciones fijas contra incendio (Tabla 1). Pero debe tenerse en cuenta que las normas IRAM no son de uso obligatorio, siendo su aplicación enteramente voluntaria a excepción de los contados casos en que alguna de estas se ha incorporado como requerimiento en normas municipales, tal el caso de las certificaciones contenidas en la familia de normas IRAM 3501 , incorporadas al Código de la Ciudad de Buenos Aires (CABA, 2019).

La ausencia de normas técnicas de aplicación obligatoria en materia de instalaciones contra incendio, sea por inexistencia de las correspondientes a la Superintendencia de Bomberos de la Policía Federal, o porque nunca se modificó la legislación vigente para convertir en obligatorio el cumplimiento de las normas IRAM implica que en muchos casos para la ejecución de instalaciones se recurra a otras normas reconocidas internacionalmente tal como las NFPA, 0 las normas armonizadas europeas $\mathrm{EN}$, muchas empleadas por requerimientos específicos de empresas, especialmente en los casos en que requieren certificar calidad en las mismas

La variedad de normas utilizadas, en razón de los diversos orígenes significa diferentes especificaciones, entre estas diferentes calidades y diámetros de tuberías y diferentes roscas lo que implica incompatibilidades críticas, tal como se mencionará más adelante.

Más aún la ausencia de normas específicas ha dado lugar a que se ejecuten instalaciones fuera de toda norma, con materiales inadecuados, si no inadmisibles, sin criterios de mantenimiento ni control lo que las deja prácticamente inoperativas, mientras dan una falsa idea de seguridad y protección a los usuarios de los edificios, lo mismo que ocurre en edificios con redes de incendio instaladas hace cincuenta o sesenta años que hace décadas que no han sido verificadas en sus condiciones de operatividad, abandono favorecido por la ausencia de normativa específica sobre control y mantenimiento de instalaciones fijas contra incendio. 


\begin{tabular}{|c|c|}
\hline IRAM 3501-1 & Certificación de la instalación. \\
\hline IRAM 3501-2 & Certificación de los auditores. \\
\hline IRAM 3501-3 & Certificación de empresas diseñadoras de instalaciones fijas contra incendios. \\
\hline IRAM $3501-4$ & Certificación de empresas instaladoras de sistemas fijos contra incendios. \\
\hline IRAM 3529 & Instalaciones fijas contra incendio. Tanques de agua. \\
\hline IRAM 3528 & Evaluación del riesgo por el método de Pourt, para sistemas automáticos de detección y extinción. \\
\hline IRAM 3531 & Sistemas de detección y alarma. Definiciones y descripción de detectores. \\
\hline IRAM 3539 & Gabinetes metálicos para mangas de incendio. Requisitos generales y ensayos. \\
\hline IRAM 3546 & Servicio de mantenimiento de instalaciones fijas contra incendios \\
\hline IRAM 3551 & Sistemas de detección y alarma. Aplicaciones \\
\hline IRAM 3552 & Detector de temperatura, puntual \\
\hline IRAM 3554 & Sistemas de detección y alarma. Proyecto y montaje de la instalación. \\
\hline IRAM 3555-1 & Sistemas de rociadores automáticos de agua. Prescripciones generales \\
\hline IRAM 3555-2 & Sistemas de rociadores automáticos de agua. Componentes. \\
\hline IRAM 3555-3 & Sistemas de rociadores automáticos de agua. Ubicación de los rociadores. \\
\hline IRAM 3555-9 & Sistemas de rociadores automáticos de agua. Inspección, ensayo y mantenimiento. \\
\hline IRAM 3556 & Sistemas de extinción. Dispositivos eléctricos de control \\
\hline IRAM 3558 & Sistemas de detección y alarma. Tableros de control y señalización \\
\hline IRAM 3577 & Sistemas de detección y alarma. Métodos de ensayo prácticos con fuego para el control de la instalación. \\
\hline IRAM 3582 & Detectores de humo, por ionización, por luz difusa y por luz transmitida. \\
\hline IRAM 3596 & Instalaciones fijas contra incendio. Rociadores automáticos. \\
\hline RAM 3597 & Instalaciones fijas contra incendios. Sistemas de hidrantes y bocas de incendio. \\
\hline IRAM 3619 & Evaluación técnica de instalaciones fijas contra incendios. \\
\hline IRAM 3632 & Sistemas de extinción a base de dióxido de carbono ( $\mathrm{CO} 2)$. \\
\hline IRAM 3635 & Sistemas de extinción a base de halon 1301. \\
\hline IRAM 3636 & Sistemas fijos de agua fraccionada. \\
\hline IRAM 3639 & Sistemas de detección y alarma. Inspección periódica. \\
\hline IRAM 3651 & Sistemas de espuma de baja expansión y de mezclas. \\
\hline IRAM 3654 & Instalaciones fijas contra incendio. Alarmas contra incendio. Para uso residencial. \\
\hline IRAM 3657-1 & Detectores de gases combustibles y mezclas explosivas. Prescripciones generales. \\
\hline IRAM 3659 & Instalaciones fijas contra incendio. Detectores de llama. \\
\hline IRAM 3668-1 & Sistemas fijos de extinción por polvo. Requisitos generales. \\
\hline IRAM 3668-2 & Sistemas fijos de extinción por polvo. De inundación total. \\
\hline IRAM 3668-3 & Sistemas fijos de extinción por polvo. De aplicación local. \\
\hline IRAM 3668-4 & Sistemas fijos de extinción por polvo. De mangas manuales. \\
\hline
\end{tabular}

Fuente: Instituto Argentino de Normalización y Certificación

Mientras existe este vacío en materia de prevención de incendios en edificios, tampoco existe en el país un marco normativo que determine los requerimientos a nivel urbano para prevenir incendios. No hay ni siquiera un esbozo, una hoja de ruta, que plantee qué tipo de servicios de bomberos o que equipamiento deben disponer estos en función del emplazamiento, el volumen construido o el tipo de actividad económico de una localidad. 


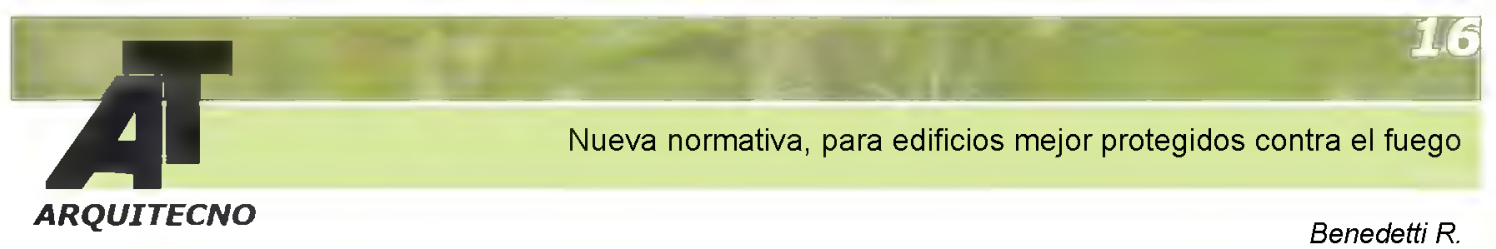

Son contados los cuerpos de bomberos profesionales existentes en las ciudades y pueblos argentinos, la enorme mayoría son cuerpos voluntarios, que se equipan con el material que pueden, cuando la ocasión lo permite. En los últimos años, gracias a la reposición obligatoria de equipos requerido por las normas europeas, los camiones norteamericanos de segunda mano que solían constituir el recurso principal de éstos se han visto complementados con camiones holandeses, alemanes y e incluso británicos (originalmente con volante a la derecha, modificados artesanalmente), muchos adquiridos con su equipamiento de origen (Figura 1).

Así, a aquella diversidad de secciones, roscas y acoples, resultado de la ausencia de normas sobre instalaciones, se adiciona otro problema, que es la diferencia de diámetros, secciones, tipos de acoples, pasos de rosca e incluso voltajes de los equipos de bomberos, que se ven limitados así en su capacidad de interoperabilidad, en un escenario que se complica aún más por la ausencia de uniformidad en las instalaciones contra incendio que eventualmente pueden llegar a encontrar en sus intervenciones.

Vale como ejemplo extremo una boca de incendio dispuesta para alimentar la red interna de una estación de servicio, la cual probablemente en cumplimiento de las especificaciones de seguridad de la empresa petrolera, fue equipada con acople tipo Storz (Figura 2), cuando lo habitual en el empleo de los bomberos locales es el empleo de conexiones provistas de rosca BSP. Como resultado, para asegurar una posibilidad de interacción exitosa entre vehículos de diferente procedencia o entre diferentes cuerpos de bomberos, y todavía con las instalaciones fijas, los bomberos se ven obligados a proveerse de antemano con un repertorio de elementos adaptadores adicionales para realizar acoples y conexiones. 
Consecuencia de esta ausencia de infraestructura -que ocurre por falta de legislación específica sobre el tema- los bomberos se ven compelidos a proveerse el recurso, para sostener las operaciones más allá de el volumen de agua transportado en la autobomba, de manera que además de organizar la extinción deben hacerse cargo de la logística para proveérsela. En las ciudades grandes cooperan en esta tarea los municipios o las empresas de servicio de agua potable, pero en los pueblos o ciudades más pequeñas deja a los bomberos a cargo de toda la operación lo que los obliga a multiplicar esfuerzos y a dividir recursos. Así no sólo deben procurarse, mantener y operar autobombas, escaleras o hidroelevadores, sino que se ven obligados a contar con camiones cisterna que permitan extender el volumen de agua a emplear, una práctica que en Estados Unidos o Europa sólo aplica en caso de fuegos estructurales en ubicaciones alejadas de los centros urbanos.

Nuestra ausencia de normativa específica y la falta de actualización de la escasa normativa que por defecto resulta aplicable en materia de seguridad edilicia y prevención de incendio, ocasiona un escaso interés en aplicar soluciones en la materia. Las columnas secas (dry riser o dry standpipe) que configuran el medio más económico para facilitar las tareas de extinción en el interior de edificios, y que en la actualidad son en casi todo el mundo un requerimiento básico para edificios en altura, localmente prácticamente no ha sido contempladas, y en cambio siguiendo al decreto 351 se sigue exigiendo -en los escasos casos en que aplica- una reserva de incendio o mixta (incendio-sanitaria) en momentos en que las consideraciones sobre conservación del agua desaconsejan acumular miles y miles de litros a la espera de una elusiva oportunidad de empleo en edificios de bajo riesgo de incendio.

Los rociadores automáticos, que en el mundo se han convertido en las más difundidas instalaciones fijas en locales y edificios de empleo comercial -y en algunos sitios, en todos los edificios en altura- en nuestro medio se mantienen limitados a casos muy específicos, o instalaciones industriales.

Aún en las cocheras, donde su uso es ideal por permitir una respuesta automática en espacios de bajo nivel de vigilancia, se mantienen obligatorios en general sólo más allá del segundo subsuelo. $Y$ no es porque episodios recientes no hayan confirmado el nivel de peligrosidad de un fuego descontrolado en un espacio de guarda de automotores, que siempre redunda en alto nivel de daños e incluso en grave afectación estructural. Aquí, al igual que con los edificios, debe tenerse en cuenta lo que ha variado la construcción automotriz, que ahora abunda en materiales sintéticos y plásticos además de contar con circuitos que permanecen permanentemente energizados.

Aun menos interés se ha suscitado por otras tecnologías, como generadores de niebla a alta presión, generadores de espuma, o de rociadores de muy alta presión, que prácticamente no han tenido ninguna aplicación local. Tampoco sistemas mucho más antiguos como la extinción automatizada por $\mathrm{CO} 2$ o HCFC-123 o incluso las instalaciones fijas de generación de espuma por AFFF, altamente capaces para situaciones específicas. Este vacío en la legislación obliga que todo proyecto que proponga emplear cualquiera de estos sistemas deba ser tratado como una excepción. O eventualmente, como ha sucedido, que se proponga su instalación como complemento de la prevista por ley, algo que no es infrecuente en la habilitación de data centers. Cabe mencionar la pertinencia del sistema mencionado de rociadores a alta presión para su aplicación en edificios de valor patrimonial, ya que la mínima sección de tuberías, la baja cantidad de agua empleada y la eficiencia en la extinción los hace particularmente indicados (Figura 3). Sin embargo, en nuestro medio su aplicación debería argumentarse en un extenso expediente de excepción, y su adquisición e instalación hacerse mediante proveedores extranjeros. En efecto, al momento de escribir este artículo no se ha podido ubicar ningún instalador local para este tipo de sistema. 

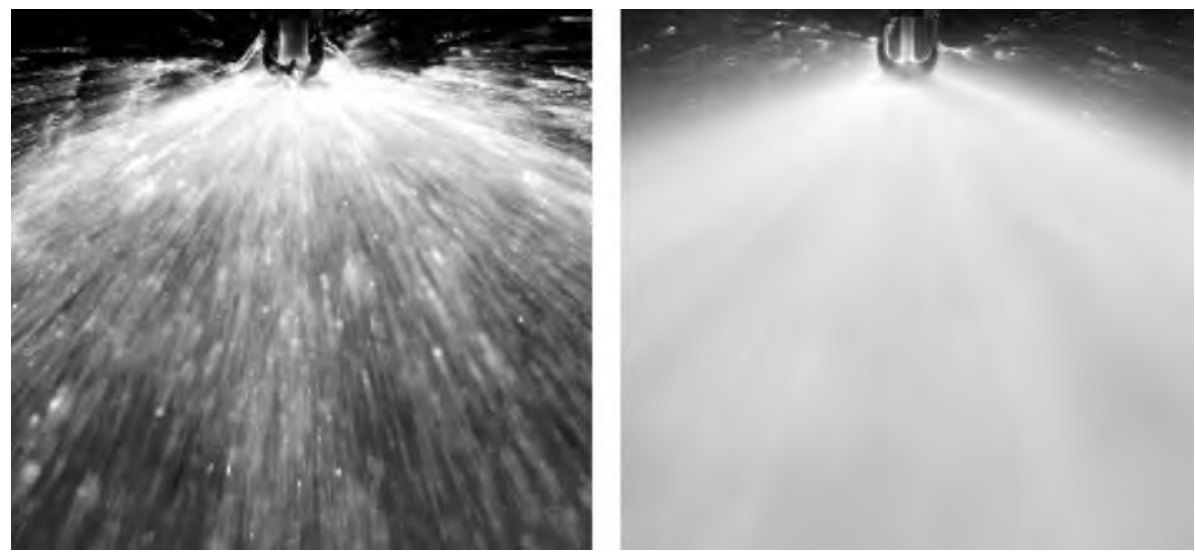

Figura 3: Comparación de proyección de agua entre un rociador estándar (izq.) y una tobera de niebla (der). Foto: HSC Seguridad.

En el contexto de la escasez normativa -escasas exigencias, ausencia de posibles solucionesse produce un nefasto doble circulo vicioso: ante la falta de obligación (y la posibilidad de lograr excepciones) la demanda de estos sistemas es baja, como la demanda es baja la oferta de proveedores e instaladores es escasa, y ante la escasa oferta los precios son elevados, desalentando más aún la demanda. Respecto a los sistemas más modernos, como no está prevista la posibilidad de empleo -salvo a través de engorrosos procesos de propuestas sustitutas- tampoco hay instaladores ni proveedores, lo que desalienta incorporarlos como solución alterna en el proyecto. Y como nadie los propone no entran nunca en consideración para actualizar la normativa.

Descripta así a grandes trazos la realidad de las medidas y los recursos contra incendio disponibles en nuestras ciudades, debemos preguntarnos qué es lo que hará falta para que la sociedad reclame mejoras sustantivas tanto en lo que se exige a los edificios como lo que ofrece la infraestructura urbana para facilitar la extinción de incendios. Las sonadas tragedias como las de República Cromañon o la explosión de Salta 2141 en Rosario solo motivaron cambios menores sin que las normativas de protección contra incendio hayan sido modificadas en profundidad. La preocupante multiplicación de incendios en edificios de departamentos, que se observa en las principales ciudades del país tampoco parece haber disparado el interés en el tema ni a nivel nacional, ni provincial ni municipal.

$Y$ no es que no existan fuentes teóricas para abrevar. Extensas y minuciosas normas extranjeras como las de la National Fire Protection Asociation (NFPA, 2018a; NFPA 2018b) que combinadas establecen las condiciones de los servicios de emergencia, la protección contra incendios y los principios para la protección de la vida en edificios nuevos y existentes proveen una referencia insoslayable tomar como base para una norma local. No obstante, es evidente que tanto los sistemas constructivos y -sobre todola disponibilidad de recursos en nuestro país difieren sustancialmente con los de los estados Unidos. Entonces, si la normativa norteamericana pudiera tomarse como demasiado inalcanzable puede optarse por las normas europeas, en tal sentido las españolas parecen accesibles y más próximas por las culturas constructivas involucradas (AENOR, 2018).

Es evidente que si en nuestro medio, si no se introducen cambios en la normativa, aumentando las exigencias en materia de detección y extinción, sólo veremos un sostenido aumento en el número de siniestros, implicando cuantiosos daños materiales y pérdida de vidas. Por otra parte, la ausencia de infraestructura y equipamiento a nivel urbano implicará que ante un stock edilicio mayor y con edificios más grandes y más 
altos, y con instalaciones cada vez más complejas, con mayor requerimiento energético, los bomberos enfrentarán cada vez mayores complicaciones para encarar las acciones de extinción. Un primer paso, elemental, pero positivo, podría darse incorporando en las normas municipales la necesidad de dar cumplimiento a las normas IRAM referidas a instalaciones fijas contra incendio en edificios construidos y a construirse. Sin embargo, esto debería complementarse con la necesidad de legislar sobre la necesidad de contar con redes de hidrantes eficaces y apropiadamente mantenidas, y la estandarización de equipos y recursos de los cuerpos de bomberos, tarea que parece más que compleja por cuanto implica establecer el alcance de la responsabilidad del Estado en esta materia. Con todo, el tema parece no concitar interés de los legisladores ni figura en la agenda pública. Esta realidad únicamente podrá revertirse si la sociedad toma conciencia que el incendio no es un riesgo lejano, sino que, al contrario, gracias al envejecimiento del stock edificado, el aumento de instalaciones energizadas, y al cambio progresivo de la cultura constructiva dominante, es una amenaza cada vez más patente, y el único resultado esperable implica incendios más frecuentes y de consecuencias más graves, con mayores daños estructurales y aumento en el número de víctimas. Quizás deban ser los profesionales involucrados en el proyecto y la construcción, y los actores del mercado inmobiliario los que deban advertir sobre este déficit, a la luz de las responsabilidades que implica respecto a terceros la construcción de inmuebles poco preparados para preservar la vida de los usuarios.

\section{BIBLIOGRAFIA}

American National Standard Institute ANSI (2018): AWWA C502 Dry-barrel fire hydrants; Washington, American Water Works Association.

Asociación Española de Normalización AENOR (2018). UNE 23500 - Sistemas de abastecimiento de agua contra incendios

Ciudad Autónoma de Buenos Aires (2019). Código de Edificación. Disponible online en: https://documentosboletinoficial.buenosaires.aob.ar/publico/PL-LEY-LCABA-LCBA-610018-ANX.pdf

Municipalidad de Rosario; Reglamento de Edificación. Disponible online en: https://www.rosario.qob.ar/mr/normativa/realamento-de-edificacion/front-paqe

National Fire Protection Asociation NFPA (2016): NFPA 1901 - Standard For Automotive Fire Apparatus.

National Fire Protection Asociation NFPA (2018 a): NFPA 1 - Fire Code

National Fire Protection Asociation NFPA (2018 b): NFPA 101 - Life Safety Catalog

República Argentina, Poder Ejecutivo Nacional. Decreto 351/1979; Buenos Aires, 5/2/1979 